\title{
Candida gut colonization, yeast species distribution, and biofilm production in Clostridioides difficile infected patients: a comparison between three populations in two different time periods
}

\author{
Grazia Brunetti ${ }^{1}$ [D - Alessandro Giuliani ${ }^{2}$ - Anna Sara Navazio ${ }^{3}$. Camilla Paradisi ${ }^{1}$. Flavia Raponi ${ }^{1}$. \\ Libenzio Adrian Conti ${ }^{1,4}$ (D) Giammarco Raponi ${ }^{1}$ (1)
}

Received: 16 October 2020 / Accepted: 27 April 2021 / Published online: 15 July 2021

(c) The Author(s) 2021

\begin{abstract}
Candida gut colonization and yeast biofilm production capacity were investigated, by means of XTT reduction assay, in Clostridioides difficile infected (CDI) patients, in non-CDI diarrheic patients, and in healthy donors in two different time periods (2013-2015 and 2018-2019 respectively). Candida gut colonization was significantly $(p<0.001)$ associated to $C$. difficile infection, and to patients infected with hypervirulent $C$. difficile strains bearing the $t c d C$ deletion at nucleotide 117 $(p=0.0003)$. Although there was not a prevalent yeast species in CDI patients, $C$. albicans was the species significantly $(p<0.001)$ associated to both the infections sustained by the non-hypervirulent $C$. difficile strains and those caused by the hypervirulent strain $(p=0.001)$. The biofilm production by the yeasts isolated from the CDI patients and from non-CDI diarrheic patients did not differ significantly. However, a significantly $(p=0.007)$ higher biofilm production was observed in the Candida strains, particularly $C$. albicans, isolated from healthy donors compared to that of the yeasts cultured from CDI patients. Seasonal occurrence was observed in the isolation rate of CDI and non-CDI diarrheic cases $(p=0.0019)$, peaking in winter for CDI patients and in spring for non-CDI diarrheic patients. Furthermore, seasonality emerged in the gut colonization by Candida of CDI patients in the winter. It seems, therefore, that the reduced capacity of biofilm production by Candida strains isolated from CDI patients might have a role in the development of $C$. difficile infection, probably facilitating the spread of the bacteria into the gut thus amplifying their pathogenic action.
\end{abstract}

Keywords Candida $\cdot$ Clostridioides difficile $\cdot$ Candida albicans $\cdot$ Biofilm $\cdot$ Seasonality

\section{Introduction}

Candida and Clostridioides difficile are two opportunistic pathogens residing in the human gut. $C$. difficile is a Gram-positive, obligate anaerobic, an endospore-forming bacterium that is one of the causes of infectious diarrhea developing in rapid spread, mostly associated with the use of broad-spectrum antibiotics and antacid therapy. The infection frequently occurs in older age hospitalized patients, in residents of long-term care facilities, in subjects suffering from immune-compromisation, malignancies, inflammatory diseases of the gastrointestinal mucosa, and previous $C$. difficile infection (CDI). Concomitantly with the disruption of the normal gut microbioma, $C$. difficile colonizes the large intestine producing the toxins primarily responsible for the associated symptoms [1]. Candida spp. and in particular C. albicans can be part of the gut microbiome of healthy individuals but the clinical relevance of such evidence is 
not known, appearing mostly to be commensals and nonpathogenic, unless the host defense system is compromised [2]. Several studies reported an association between CDI and candidemia [3, 4], and recent CDI was one of the factors independently associated with candidemia [5]. The common pathogenetic pathway could be found on the alteration of the human microbiome induced by antimicrobial therapy, which might favor both CDI and persistence of Candida in the gastrointestinal (GI) tract [6]. In turn, the extensive mucosal damage caused by the toxins produced by $C$. difficile might allow the dissemination of the yeast in the bloodstream. The virulence of $C$. albicans depends on the growth in yeast, pseudohyphal, and hyphal forms and to the capacity to survive in a biofilm state. The latter could be a phenomenon of paramount importance since the microenvironment of $C$. albicans biofilms can favor the growth of anaerobic microorganism such as Clostridium perfringens under normally toxic aerobic conditions [7]. In a previous study, we evidenced a link between CDI and Candida colonization of the gut [8]. Nowadays, different authors evidenced either positive or negative correlation between CDI and Candida. Since the debate is still open, in this study, we investigated the differences in the Candida gut colonization of CDI patients, of non-CDI diarrheic patients, and of healthy donors in two different periods with different epidemiological CDI diffusion. Furthermore, we wanted to investigate whether the metabolically active biofilm production by Candida differed among strains isolated from the three study populations.

\section{Materials and methods}

In this control study, 482 stool specimens were sampled at the 1300 beds-teaching hospital Policlinico "Umberto I" in Rome, Italy. The study analyzed existing laboratory data that were anonymized before being included in the study database. Stool specimens were collected at the hospitalization site, placed at $4{ }^{\circ} \mathrm{C}$ in the absence of preservative substances, and processed within $30 \mathrm{~min}$ from the collection. Diarrheic stool specimens were defined as $\geq 5$ of the Bristol stool chart [9], considering a rejection criterion if the score was $<5$, except in the case of specimen collection from healthy donors. In order to compare homogenous populations, 243 consecutive diarrheic stool specimens were collected between November 2013 and March 2015, as well as 239 stool specimens, including 106 stool samples from healthy donors and 133 diarrheic stool specimens positive for the presence of toxinogenic $C$. difficile, in the period between May 2018 and May 2019. All the stool samples were investigated for the presence of toxinogenic $C$. diffcile following a two steps diagnostic algorithm, in which all the samples displaying positive reaction to the immunechromatographic GDH assay screening (C. diff quik chek®,
TechLab, Blacksburg, USA) were analyzed for the presence of toxin B ( $t c d B$ gene), binary toxin ( $c d t A$ gene), and a $t c d C$ deletion gene at nucleotide 117 (NAP-BI-027 presumptive positive), by RT-PCR (Xpert ${ }^{\circledR}$ C. difficile, Cepheid, Sunnyvale, USA). Quantification of Candida gut colonization of all the fecal samples was achieved by diluting $10 \mu \mathrm{L}$ of diarrheic stool sample or a net loop of solid stool sample (approx. $10 \mu \mathrm{g}$ ) in $1 \mathrm{ml}$ of sterile $0.9 \% \mathrm{NaCl}$ solution, plating $10 \mu \mathrm{L}$ on Sabouraud dextrose agar supplemented with chloramphenicol (Oxoid spa, Milan, Italy). Plates were incubated for at least $24 \mathrm{~h}$ and up to 5 days at $30{ }^{\circ} \mathrm{C}$. Yeast growth was defined as positive if $\geq 10^{4} \mathrm{CFU} / \mathrm{ml}$ grew from the stool samples [2]. The yeasts were typed by matrix-assisted laser desorption/ionization assay (MALDI-TOF Bruker Daltonik $\mathrm{GmbH}$, Bremen, Germany), using an extraction process by means of ethanol treatment, followed by formic acid and acetonitrile extraction and accepting score values $\geq 1.8$ $[10,11]$. Yeast strains were frozen and stored at $-80^{\circ} \mathrm{C}$ in Microbank ${ }^{\mathrm{TM}}$ Storage system (Pro-Labs Diagnostics, Richmond Hill, Ontario, Canada) until analysis. The formation of metabolic active fungal biofilm of Candida strains was measured in vitro by means of 2,3-bis (2-methoxy-4-nitro-5-sulfophenyl)-2H-tetrazolium-5-carboxanilide (XTT, Sigma-Aldrich s.r.l., Milan, Italy) reduction assay with slight modifications [12]. Briefly, each Candida strain in the logphase of growth $\left(200 \mu \mathrm{l}\right.$ of $10^{6}$ cells/ml in RPMI 1640) was plated on flat-bottomed 96 -well polystyrene microtiter plates and incubated at $37{ }^{\circ} \mathrm{C}$ for $48 \mathrm{~h}$. After incubation, the plates were washed with sterile PBS and added with $100 \mu \mathrm{L}$ of a solution $0.67 \mathrm{~g} / \mathrm{L}$ XTT-1 $\mu$ M menadione (Sigma-Aldrich). The resulting color change was spectrophotometrically quantified at $490 \mathrm{~nm}$ with a microtiter plate reader (Eti System Reader, Bio-Tek Instruments Inc., Winooski, VT, USA). To standardize the method, the results were expressed in terms of Biofilm Index (BI) [13] calculated as the ratio between the absorbance test strain and the absorbance of a stable biofilm-producing Candida albicans strain (C. albicans SA40, kind gift from dr. F. De Bernardis, National Health Institute of Rome) [14]. Three different categories of patients, i.e., patients carrying toxinogenic $C$. difficile (CDI), nonCDI diarrheic patients, and healthy donors, were considered in two different time intervals (years 2013-2015 vs. 2018-2019). Informed written patients' consent was not required because of the observational nature of the study. The Ethical Committee of "Sapienza" University of Rome (Prot. 13/18) approved the study.

\section{Statistical analysis}

The production of biofilm by the yeast isolates was expressed as the mean \pm SD of the BI value. Statistical analyses were performed using the SAS/STAT software. We adopted a chisquare correlation metrics when in presence of categorical 
variables; thus, inferential analysis was based on $\chi^{2}$ test or Fisher's exact test when needed. Inferential statistics on continuous variables was performed by means of Student's $t$ test for two group comparisons and by analysis of variance (ANOVA) when in presence of more than two groups. An $\alpha$ error $<0.05$ was accepted as significance threshold.

\section{Results}

\section{Clostridioides difficile in diarrheic patients}

In this case-control study, 482 stool specimens were collected. On basis of year isolation (2013-2015 vs. 2018-2019), these samples were analyzed and compared. The statistical analysis confirmed that the two studied temporal phases were homogeneous by the relative percentage of the number and age of patients' distribution, the month of isolation, and the number of Candida strains tested for biofilm production capacity. In the years 2013-2015, diarrheic stool specimens $(\mathrm{nr}=243)$ were investigated for the positivity to $C$. difficile. Among these samples, 136 derived from non-CDI diarrheic patients (median age $61 \pm 22.41$, $44.12 \%$ female), while 107 resulted positive to the RT-PCR test. Of these, 70 patients suffered from non-hypervirulent CDI (median age $70 \pm 18.01,60 \%$ female), and $34.58 \%$ displayed the $t c d C$ deletion at nucleotide 117 (NAP-BI-027 presumptive positive, median age $56.76 \pm 15,56.76 \%$ female). In 2018-2019, 239 stool specimens were analyzed: 133 samples tested positive to RT-PCR, 105 resulted positive to nonhypervirulent $C$. difficile (median age $71 \pm 17,49.1 \%$ female) with $21 \%$ positivity for hypervirulent $C$. difficile (median age $75.8 \pm 18.01,60 \%$ female), and 106 samples were derived from healthy donors (median age $50 \pm 20,50 \%$ female). An epidemiological shift of the toxinogenic profile of CDI diffusion was observed among the two periods. Indeed, a statistically significant increase in the prevalence of the nonhypervirulent strains (i.e., strains not bearing the $t c d C$ gene deletion at nucleotide 117) was observed in 2018-2019 compared to 2013-2015 (chi-square $=5.49, p=0.01$ ). Moreover, a higher incidence of infections sustained by $C$. difficile was confirmed in female (chi-square $=5.21, p=0.02$ ) and elderly patients (median age $=71.40$; chi-square 25.74, $p<0.001$ ). The age was significantly higher in hypervirulent $C$. difficile than non-hypervirulent $C$. difficile patients (74.76 vs. 65.91 , respectively) $(p<0.001)$.

\section{Clostridioides difficile and Candida colonization}

Candida colonization of the gut was investigated in all 482 patients and found in 288 samples. Data analysis showed that Candida colonization varied in the different group of patients. In fact, while Candida colonization rate was of
$25.47 \%$ in the healthy donors group $(\mathrm{nr}=106)$, the general rate observed in all diarrheic stool samples, collected from both CDI patients and non-CDI diarrheic patients, approached $69 \%$ in both groups. Considering the data in the different phases of the study (2013-2015 vs. 2018-2019), it emerged that in the first period, Candida colonized the intestinal gut of $90.65 \%$ of CDI patients $(\mathrm{nr}=107)$ and of $69.12 \%$ of non-CDI diarrheic patients $(\mathrm{nr}=136)$. This relative frequency difference was statistically significant (chi-square $=16.51, p<0.001)$ indicating a strong specific association between Candida colonization and CDI. Furthermore, in the second period when comparing Candida gut colonization of CDI patients and of healthy donors, this association was statistically confirmed (chi-square $=18.04$, $p<0.001$ ) (Fig. 1). In fact, while in healthy donors, Candida colonized only $25.47 \%$ of 106 enrolled patients, in CDI patients, Candida gut colonization was observed in 52.63\% of the 133 samples. Analyzing the whole set of patients and comparing the three different categories of patients, Candida gut colonization was confirmed significantly associated to CDI (chi-square $=66.4, p<0.0001$ ). Furthermore, this association was also confirmed when studying only the subgroup of patients infected with $C$. difficile strains bearing the $t c d C$ gene deletion at nucleotide 117 (chisquare $=12.81, p=0.0003$ ). Stratifying the data according to the species of colonizing Candida in the overall analysis, C. albicans was the most prevalent species (58.7\%), followed by $C$. glabrata, both in CDI patients and in non-CDI diarrheic patients ( $52.1 \%$ and $22 \%$, respectively). A similar situation was observed in the group of healthy donors in which C. albicans $(59.2 \%)$ was the species most frequently isolated followed by $C$. glabrata $(18.5 \%)$. The distribution of Candida species in the different periods and in the different groups was homogeneous, witnessing that there was no species prevalence in the different groups. However, when the statistical analysis included all the enrolled patients, it emerged that Candida albicans was the species most frequently associated with CDI both in the analysis between CDI and non-CDI diarrheic patients (chi-square $=22.12$, $p=0.0005)$ and between CDI patients and healthy donors (chi-square $=20.9177, p=0.0008)$ and in the overall analysis (chi-square $=80.86, p<0.001)$ (Tables 1 and 2). This association was also reflected in the correlation between $C$. albicans colonization in patients infected by the $C$. difficile strains bearing the $t c d C$ gene deletion at nucleotide 117 (hypervirulent) (chi-square $=20.47, p=0.001$ ).

\section{Seasonality}

Differential distribution of cases among CDI patients and non-CDI diarrheic patients was observed stratifying data according to the months of isolation (chi-square $=14.86$, $p=0.0019$ relative to non-CDI-diarrheic patients). In 
Fig. 1 Association between Candida colonization and CDI infections. The statistical association emerged when comparing the percentages of Candida gut colonization in CDI patients and in non-CDI diarrheic patients (chi-square $=16.51, p<0.001$ ), as well as when comparing the percentages of colonization in CDI patients and in healthy donors (chi-square $=18.04$, $p<0.001)$

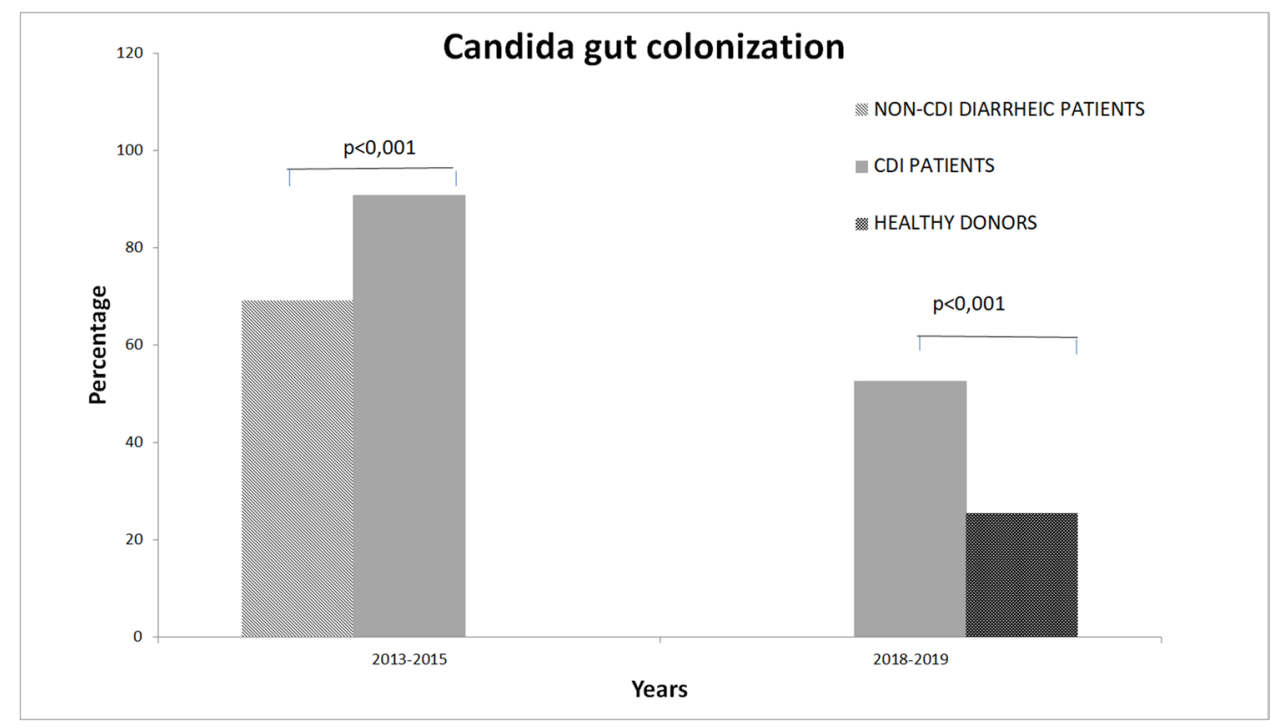

particular, in the first period of study, the highest CDI isolation rate was observed during the winter, while nonCDI diarrheic cases peaked in spring and the relative distribution of cases was specular. While during autumn, the percentage of episodes was relatively uniform, in the summer, the number of events involving $C$. difficile was lower (Fig. 2). Moreover, Candida gut colonization in CDI patients evidenced seasonality as well. In fact, despite the percentage of Candida colonization remained steady in the various seasons, the analysis of the relative yes/no distribution showed that the highest percentage of $\mathrm{Can}$ dida colonization reached $88.68 \%$ in winter and $82.00 \%$ in spring $($ chi-square $=22.22, p<0.0001)($ Table 3$)$.

Table 1 Gut colonization by Candida species. In the overall analysis, Candida albicans was the species most frequently associated with CDI (chi-square $=80.86, p<0.001)$. ${ }^{*}$ Other species included $C$. guilliermondii, C. krusei, C. sake, and C. lusitaniae. In parenthesis are reported the number of cases

\begin{tabular}{|c|c|c|c|}
\hline \\
\hline \multicolumn{4}{|c|}{$\begin{array}{ll}\text { CDI patients } & \begin{array}{l}\text { Non-CDI } \\
\text { diarrheic } \\
\text { patients }\end{array}\end{array}$} \\
\hline Candida albicans & $40.83 \%(98)$ & $36.03 \%(49)$ & $15.09 \%(16)$ \\
\hline Candida glabrata & $15.42 \%(37)$ & $11.03 \%(15)$ & $4.72 \%(5)$ \\
\hline Candida parapsilosis & $1.25 \%(3)$ & $2.94 \%(4)$ & $1.89 \%(2)$ \\
\hline Candida tropicalis & $4.58 \%(11)$ & $2.21 \%(3)$ & $0.94 \%(1)$ \\
\hline Other* & $7.50 \%(18)$ & $16.91 \%(23)$ & $2.83 \%(3)$ \\
\hline $\begin{array}{l}\text { No Candida coloniza- } \\
\text { tion }\end{array}$ & $30.42 \%(73)$ & $30.88 \%(42)$ & $74.53 \%(79)$ \\
\hline
\end{tabular}

\section{Biofilm production}

The biofilm production was performed on 234/288 Candida strains that were viable after thawing (140 isolated from the stools of CDI positive patients, 67 Candida strains isolated from non-CDI diarrheic patients, and 27 Candida strains isolated from healthy donors) through XTT reduction assay, equating the percentage of the Candida species distribution. Results were expressed in terms of Biofilm Index (BI). From the comparison between the three studied patients' categories, no statistically significant differences emerged in the production of $\mathrm{BI}$, as well as in the comparison between CDI patients and non-CDI diarrheic patients. However, the statistical analysis showed a higher biofilm production by Candida strains isolated from healthy donors compared to CDI patients ( $p=0.007)$ (Fig. 3). C. albicans had the highest capacity in producing biofilm compared to the other Candida species. However, there was no higher biofilm production by $C$. albicans isolated from CDI patients compared to that of non-CDI diarrheic patients. Instead, C. albicans biofilm production was significantly enhanced when the strains were isolated from healthy donors compared to CDI patients $(p=0.0006)$. This increase was not observed when biofilm production capacity was measured in strains of $\mathrm{Can}$ dida albicans isolated from healthy donors compared to the biofilm production in non-CDI diarrheic patients.

\section{Discussion}

It is widely accepted that antibiotic-induced alteration of the intestinal flora might have a positive effect on Candida spp. gut colonization. Several studies have focused on the prevalence of $C$. albicans in $C$. difficile infected patients, yet the 
Table 2 Gut colonization by Candida species among CDI and non-CDI diarrheic patients in the period 2013-2015 and between CDI patients and healthy donors in the period 2018-2019. Candida albicans was the species most frequently associated with CDI in the two different periods (chi-square $=22.12, p=0.0005$ and chisquare $=20.9177, p=0.0008$ respectively). ${ }^{*}$ Other species included C. guilliermondii, C. krusei, $C$. sake, and C. lusitaniae. In parenthesis are reported the number of cases

\begin{tabular}{|c|c|c|c|c|}
\hline & \multicolumn{2}{|l|}{$2013-2015$} & \multicolumn{2}{|l|}{$2018-2019$} \\
\hline & CDI patients & Non-CDI diarrheic patients & CDI patients & Healthy donors \\
\hline Candida albicans & $56.07 \%(60)$ & $36.03 \%(49)$ & $28.57 \%(38)$ & $15.09 \%(16)$ \\
\hline Candida glabrata & $17.76 \%(19)$ & $11.03 \%(15)$ & $13.53 \%(18)$ & $4.72 \%(5)$ \\
\hline Candida parapsilosis & $0.93 \%(1)$ & $2.94 \%(4)$ & $1.50 \%(2)$ & $1.89 \%(2)$ \\
\hline Candida tropicalis & $2.80 \%(3)$ & $2.21 \%(3)$ & $6.02 \%(8)$ & $0.94 \%(1)$ \\
\hline Other* & $13.08 \%(14)$ & $16.91 \%(23)$ & $3.01 \%(4)$ & $2.83 \%(3)$ \\
\hline No Candida colonization & $9.35 \%(10)$ & $30.88 \%(42)$ & $47.37 \%(63)$ & $74.53 \%(79)$ \\
\hline
\end{tabular}

nature of the interactions between these microorganisms is still controversial $[15,16]$. In a previous study, we observed that CDI-positive patients had high rates of $C$. albicans gut colonization [8], and another paper reported non-albicans Candida (NAC) colonization in a trial on 548 patients with CDI [17]. Conversely, Manian et al. reported a negative association between Candida spp. overgrowth and CDI [16]. In our study, despite similar patient characteristics in two different periods, the fecal samples from patients with CDI demonstrated a consistent and significant Candida gut colonization that was not present among non-CDI diarrheic patients. Moreover, we found strong correlation between CDI and Candida spp. colonization due to the observation that the stool samples of healthy donors displayed a significantly lower incidence of Candida colonization. In particular, if we extended the study to the whole group of enrolled patients, Candida albicans was the yeast most frequently isolated in CDI patients. Nowadays, this yeast is considered a common gut commensal of humans, influencing the composition of the bacterial microbioma [18]. However, in our study, only $15.09 \%$ of the healthy donors had C. albicans colonization, though it was the species most frequently isolated $(59.2 \%)$. Therefore, we can speculate that the higher and statistically significant intestinal colonization by Candida spp. in CDI patients, and in particular by C. albicans, might be promoted by the antibiotic therapy. As widely described in the literature, the antibiotics can be considered the principal risk factor for CDI with non-specific effect on gut microbioma [19-21]. Several studies found seasonality of CDI with different peaks depending on the different geographical area [22]. Seasonal climate [23] and the correlation with other respiratory infections have been invoked to explain such findings [24, 25]. Suda et al. evidenced a higher prescription rate of antibiotics during winter [26], supporting the hypothesis that seasonal antibiotic prescriptions rate might have contributed to the seasonality of CDI observed in our study, a finding that coincided with the highest percentage
Fig. 2 Isolation rate of CDI and non-CDI diarrheic cases. Statistical association emerged in the distribution of the percentage of case (chi-square $=14.86$, $p=0.0019)$. Seasonality data were referred to four-season periods as follows: winter, January 1 until March 31; spring, April 1 until June 30; summer, July 1 until September 30; autumn, October 1 until December 31

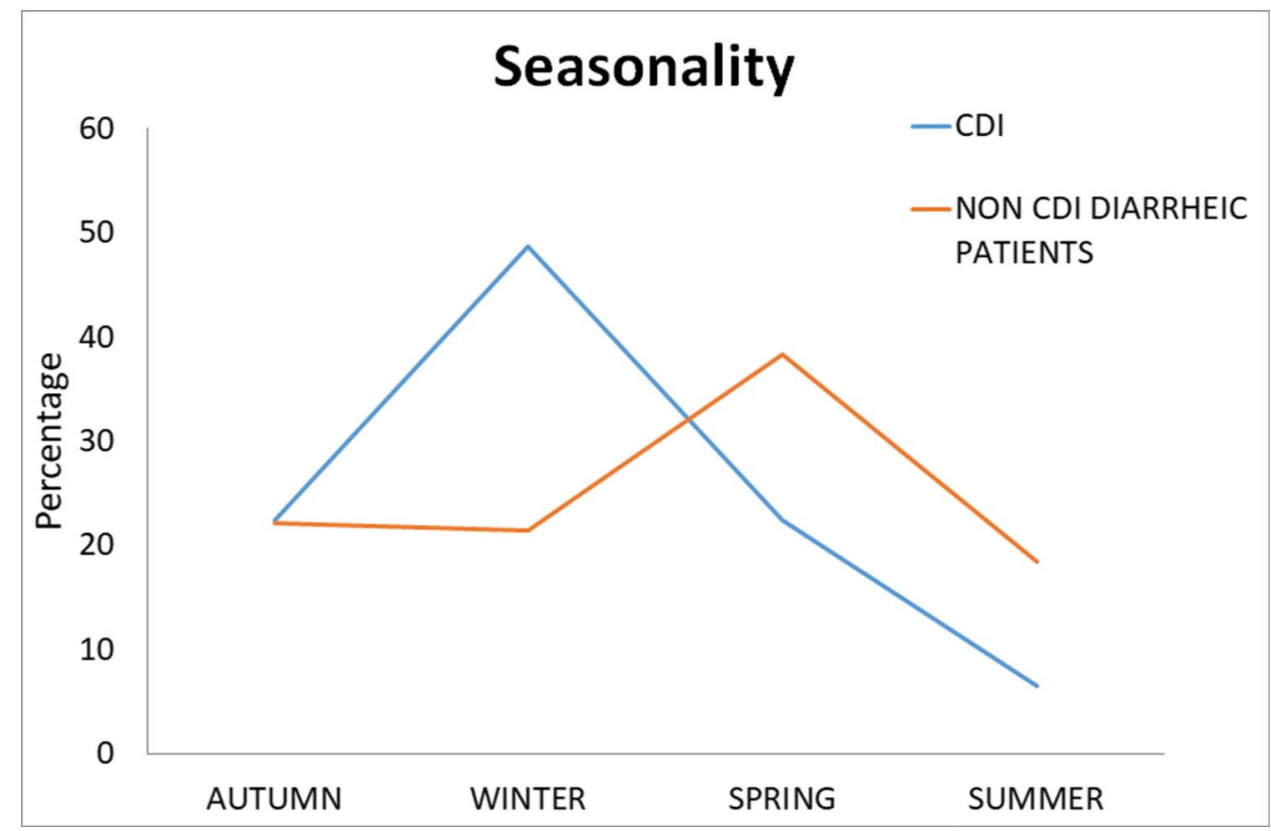


Table 3 Global analysis of the yes/no relative distribution of Candida gut colonization in CDI patients. Seasonality data were referred to four-season periods as follows: winter, January 1 until March 31; spring, April 1 until June 30; summer, July 1 until September 30; autumn, October 1 until December 31

\begin{tabular}{lll}
\hline Season & \multicolumn{2}{l}{$\begin{array}{l}\text { Candida } \text { gut colonization in CDI patients } \\
(\%, \text { number of cases })\end{array}$} \\
\cline { 2 - 3 } & No & Yes \\
\hline Autumn & $40.30 \%(27)$ & $59.70 \%(40)$ \\
Winter & $11.32 \%(6)$ & $88.68 \%(47)$ \\
Spring & $18.00 \%(9)$ & $82.00 \%(41)$ \\
Summer & $44.29 \%(31)$ & $55.71 \%(39)$ \\
\hline
\end{tabular}

of Candida gut colonization. In the interplay between $C$. difficile and $C$. albicans, the yeast might promote the development of CDI expanding the ecological niches of $C$. difficile so that its vegetative cells might be able to survive to an oxygenated environment. This hypothesis was underscored by the findings of Pim et al. who demonstrated that $C$. difficile can survive to an oxygenated environment when co-cultured with $C$. albicans [15]. The interactions between $C$. difficile and $C$. albicans could be multiple, but the capacity of the yeast to produce biofilm might be of paramount importance in the pathogenic processes of CDI leading to invasion of the mucosal cells [27]. Hence, the ability of Candida to produce biofilm could mediate $\mathrm{CD}$ adhesion to the gut, thus allowing the release of $C$. difficile toxins in the intestinal mucosa, the dissemination, and the onset of the disease. Therefore, in our study, to ascertain whether CDI was associated to Candida strains with higher biofilm-producing capacity, the biofilm production was measured in Candida strains isolated from the stools of CDI patients, of non-CDI diarrheic patients,

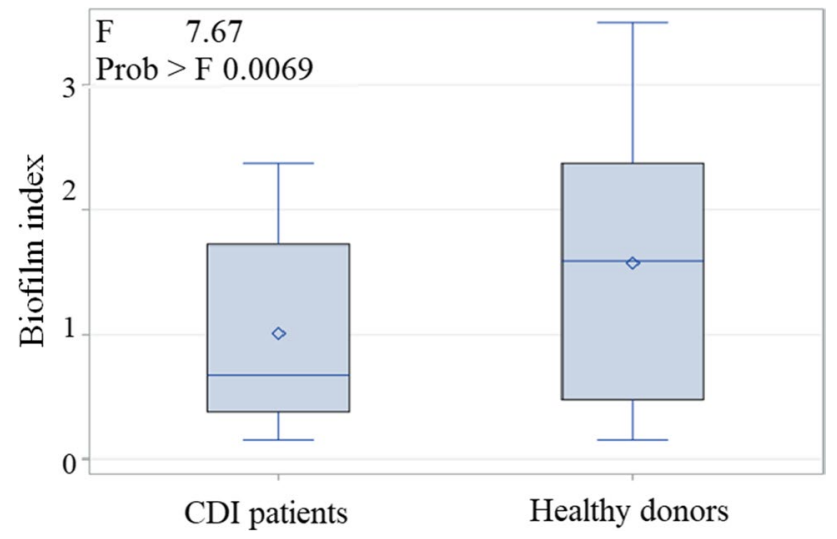

Fig. 3 Comparison of biofilm production capacity of Candida (expressed in terms of Biofilm Index) between CDI patients and healthy donors. The statistical analysis showed a higher biofilm production by Candida strains isolated from healthy donors compared to CDI patients $(p=0.007)$ and of healthy donors. No significant differences emerged in the Candida biofilm production between Candida strains isolated from CDI patients and the strains isolated from non-CDI diarrheic patients. However, a higher biofilm production by Candida strains, in particular C. albicans, was observed when the strains were cultured from healthy donors compared to that of yeasts isolated from CDI patients. It is possible that the intense host inflammatory response caused by toxin hyper production [28] and the intestinal mucosal barrier destruction [29, 30] could inhibit Candida biofilm production, thus allowing penetration of $\mathrm{CD}$ into the mucosa and manifesting its pathogenic action. Pim et al. demonstrated that, although C. albicans allowed $C$. difficile to survive in aerobic ambient, when this organism was co-cultured with $C$. albicans, it produced chemical signals such as p-cresol with inhibitory activity against the $C$. albicans biofilm formation [15]. These latter findings seem to further explain the higher incidence of C. albicans isolation in CDI patients observed in our study, together with the observation that biofilm production was not an intrinsic capacity of the Candida strain involved in the infection, rather by the greater frequency of isolation of Candida spp., in particular C. albicans, in CDI infection. Not in the end, in our study, we observed a change of the toxinogenic profile of CDI diffusion, with a significant increase in the prevalence of the non-hypervirulent $C$. difficile strains (i.e., strains not expressing the $t c d C$ gene deletion at nucleotide 117 ) in the second period of the study compared to the first one. This phenomenon could be explained by the implementation of control and containment measures of this infection applied in our hospital, and it will be the topic of forthcoming studies. Our study suffers of two major limitations. Firstly, being a retrospective analysis, there is a lack of data regarding patients' clinical features and the antimicrobial treatment received by the single patient before the development of CDI that may have influenced the Candida gut colonization. Secondly, the study lacks data regarding the clonality of both $C$. difficile and Candida isolates in order to correlate the interactions between the two species.

\section{Conclusion}

Candida gut colonization accompanied symptomatic CDI, in particular those sustained by $C$. difficile strains bearing the $t c d C$ deletion gene at nucleotide 117 . The antibiotic prescription rate could play a key role in the alteration of the intestinal flora. In the winter months, we recorded a greater percentage of Candida gut colonization, and, surprisingly, just in these months, we registered the peak of $C$. difficile infections determining the seasonality. In CDI patients, the percentage of Candida colonization was three times higher than the percentage of Candida colonization in the healthy donors, such as the $C$. albicans colonization. No single 
isolated species of Candida was prevalent in CDI patients, but when considering all the patients enrolled, the greater frequency of isolation of $C$. albicans was associated both to the non-hypervirulent CDI strains and to strains bearing the $t c d C$ gene deletion at nucleotide 117 . No statistically significant differences emerged in the biofilm production from the comparison of Candida strains isolated in healthy donors and diarrheic non-CDI patients, with the notable exception of $C$. albicans that showed an increased biofilm production when isolated from healthy donors compared to CDI patients. The interactions between $C$. difficile and $C$. albicans could be multiple and the infection could progress through various steps allowing the penetration of $\mathrm{CD}$ into the mucosa and manifesting the pathogenic action.

Funding Open access funding provided by Università degli Studi di Roma La Sapienza within the CRUI-CARE Agreement.

Data availability All relevant data are within the paper.

\section{Declarations}

Ethics approval Ethical Committee of "Sapienza" University of Rome (Prot. 13/18).

Consent to participate All authors gave formal consent to participate to the study.

Consent for publication All authors read and approved the manuscript in its present form.

Competing interests The authors declare no competing interests.

Open Access This article is licensed under a Creative Commons Attribution 4.0 International License, which permits use, sharing, adaptation, distribution and reproduction in any medium or format, as long as you give appropriate credit to the original author(s) and the source, provide a link to the Creative Commons licence, and indicate if changes were made. The images or other third party material in this article are included in the article's Creative Commons licence, unless indicated otherwise in a credit line to the material. If material is not included in the article's Creative Commons licence and your intended use is not permitted by statutory regulation or exceeds the permitted use, you will need to obtain permission directly from the copyright holder. To view a copy of this licence, visit http://creativecommons.org/licenses/by/4.0/.

\section{References}

1. Smits WK, Lyras D, Lacy DB, Wilcox MH, Kuijper EJ (2016) Clostridium difficile infection. Nat Rev Dis Primers 2:16020. https://doi.org/10.1038/nrdp.2016.20

2. Schulze J, Sonnenborn U (2009) Yeasts in the gut: from commensals to infectious agents. Dtsch Arztebl Int 106(51-52):837-842. https://doi.org/10.3238/arztebl.2009.0837

3. De Rosa FG, Corcione S, Raviolo S, Montrucchio C, Aldieri C, Pagani N, Di Perri G (2015) Candidemia, and infections by Clostridium difficile and carbapenemase-producing
Enterobacteriaceae: new enteropathogenetic opportunistic syndromes? Infez Med 23(2):105-116

4. Falcone M, Venditti M, Sanguinetti M, Posteraro B (2016) Management of candidemia in patients with Clostridium difficile infection. Expert Rev Anti Infect Ther 14(7):679-685. https://doi.org/ 10.1080/14787210.2016.1197118

5. Falcone M, Tiseo G, Tascini C, Russo A, Sozio E, Raponi G, Rosin C, Pignatelli P, Carfagna P, Farcomeni A, Luzzati R, Violi F, Menichetti F, Venditti M (2017) Assessment of risk factors for candidemia in non-neutropenic patients hospitalized in Internal Medicine wards: a multicenter study. Eur J Intern Med 41:33-38. https://doi.org/10.1016/j.ejim.2017.03.005

6. Ruiz-Sánchez D, Calderón-Romero L, Sánchez-Vega JT, Tay J (2002) Intestinal candidiasis. A clinical report and comments about this opportunistic pathology. Mycopathologia. 156:9-11. https://doi.org/10.1023/A:1021326713470

7. Fox EP, Cowley ES, Nobile CJ, Hartooni N, Newman DK, Johnson AD (2014) Anaerobic bacteria grow within Candida albicans biofilms and induce biofilm formation in suspension cultures. Curr Biol 24(20):2411-2416. https://doi.org/10.1016/j.cub.2014.08.057

8. Raponi G, Visconti V, Brunetti G, Ghezzi MC (2014) Clostridium difficile infection and Candida colonization of the gut: is there a correlation? Clin Infect Dis 59(11):1648-1649. https:// doi.org/10.1093/cid/ciu637

9. Lewis SJ, Heaton KW (1997) Stool form scale as a useful guide to intestinal transit time. Scand J Gastroenterol 32(9):920-924. https://doi.org/10.3109/00365529709011203

10. Ghosh AK, Paul S, Sood P, Rudramurthy SM, Rajbanshi A, Jillwin TJ, Chakrabarti A (2015) Matrix-assisted laser desorption ionization time-of-flight mass spectrometry for the rapid identification of yeasts causing bloodstream infections. Clin Microbiol Infect 21(4):372-378. https://doi.org/10.1016/j.cmi. 2014.11.009

11. Pinto A, Halliday C, Zahra M, van Hal S, Olma T, Maszewska K, Iredell JR, Meyer W, Chen SCA (2011) Matrix-assisted laser desorption ionization-time of flight mass spectrometry identification of yeasts is contingent on robust reference spectra. PLoS ONE. 6(10):e25712. https://doi.org/10.1371/journal.pone.0025712

12. Ramage G, Vande Walle K, Wickes BL, López-Ribot JL (2001) Standardized method for in vitro antifungal susceptibility testing of Candida albicans biofilms. Antimicrob Agents Chemother 45(9):2475-2479. https://doi.org/10.1128/aac.45.9.2475-2479. 2001

13. Brunetti G, Visconti V, Ghezzi MC, Giordano A, Raponi G. The correlation between biofilm production and catheter related blood stream infections sustained by Candida. a case control study. Adv Exp Med Biol. 2017. https://doi.org/10.1007/5584_2016_196

14. Sandini S, Stringaro A, Arancia S, Colone M, Mondello F, Murtas S, Girolamo A, Mastrangelo N, De Bernardis F (2011) The MP65 gene is required for cell wall integrity, adherence to epithelial cells and biofilm formation in Candida albicans. BMC Microbiol 11:106. https://doi.org/10.1186/1471-2180-11-106

15. Pim T., van Leeuwen J.M. van der Peet F. J., Bikker M.A. Hoogenkamp A.M., Oliveira Paiva, Sarantos K., Oleg A. Mayboroda, Wiep Klaas Smits, Krom B.P. Interspecies interactions between Clostridium difficile and Candida albicans study. mSphere. 2016; 1(6):e00187-16; https://doi.org/10.1128/mSphere.00187-16

16. Manian FA, Bryant A (2013) Does Candida species overgrowth protect against Clostridium difficile infection? Clin Infect Dis 56(3):464-465. https://doi.org/10.1093/cid/cis854

17. Nerandzic MM, Mullane K, Miller MA, Babakhani F, Donskey CJ (2012) Reduced acquisition and overgrowth of vancomycinresistant enterococci and Candida species in patients treated with fidaxomicin versus vancomycin for Clostridium difficile infection. Clin Infect Dis 55(Suppl 2):S121-S126. https://doi.org/10.1093/ $\mathrm{cid} / \mathrm{cis} 440$ 
18. Huffnagle GB, Noverr MC (2013) The emerging world of the fungal microbiome. Trends Microbiol 21:334-341

19. Loo VG, Bourgault AM, Poirier L, Lamothe F, Michaud S, Turgeon N, Toye B, Beaudoin A, Frost EH, Gilca R, Brassard P, Dendukuri N, Béliveau C, Oughton M, Brukner I, Dascal A (2011) Host and pathogen factors for Clostridium difficile infection and colonization. N Engl J Med 365(18):1693-1703. https://doi.org/ 10.1056/NEJMoa1012413

20. Furuya-Kanamori L, Stone JC, Clark J, McKenzie SJ, Yakob L, Paterson DL, Riley TV, Doi SAR, Clements AC (2015) Comorbidities, exposure to medications, and the risk of communityacquired Clostridium difficile infection: a systematic review and meta-analysis. Infect Control Hosp Epidemiol 36(2):132-41. https://doi.org/10.1017/ice.2014.39

21. Webb BJ, Subramanian A, Lopansri B, Goodman B, Jones PB, Ferraro J, Stenehjem E, Brown SM (2020) Antibiotic exposure and risk for hospital-associated Clostridioides difficile infection. Antimicrob Agents Chemother 64(4):e02169-e2219. https://doi. org/10.1128/AAC.02169-19

22. Furuya-Kanamori L, McKenzie SJ, Yakob L, Clark J, Paterson DL, Riley TV, Clements AC (2015) Clostridium difficile infection seasonality: patterns across hemispheres and continents - a systematic review. PLoS ONE 10(3):e0120730. https://doi.org/10. 1371/journal.pone.0120730

23. Furuya-Kanamori L, Robson J, Soares Magalhães RJ, Yakob L, McKenzie SJ, Paterson DL, Riley TV, Clements AC (2014) A population-based spatio-temporal analysis of Clostridium difficile infection in Queensland, Australia over a 10-year period. J Infect 69(5):447-455. https://doi.org/10.1016/j.jinf.2014.06.014

24. Polgreen PM, Yang M, Bohnett LC, Cavanaugh JE (2010) A timeseries analysis of clostridium difficile and its seasonal association with influenza. Infect Control Hosp Epidemiol 31(4):382-387. https://doi.org/10.1086/651095
25. Gilca R, Fortin E, Frenette C, Longtin Y, Gourdeau M (2012) Seasonal variations in Clostridium difficile infections are associated with influenza and respiratory syncytial virus activity independently of antibiotic prescriptions: a time series analysis in Quebec. Canada Antimicrob Agents Chemother 56(2):639-646. https://doi. org/10.1128/AAC.05411-11

26 Suda KJ, Hicks LA, Roberts RM, Hunkler RJ, Taylor TH (2014) Trends and seasonal variation in outpatient antibiotic prescription rates in the United States, 2006 to 2010. Antimicrob Agents Chemother. 58(5):2763-6. https://doi.org/10.1128/AAC.02239-13

27. Sudbery PE (2011) Growth of Candida albicans hyphae. Nat Rev Microbiol 9:737-748. https://doi.org/10.1038/nrmicro2636

28. Madan R, Petri WA Jr (2012) Immune responses to Clostridium difficile infection. Trends Mol Med 18(11):658-666. https://doi. org/10.1016/j.molmed.2012.09.005

29. Nam HJ, Kang JK, Kim SK, Ahn KJ, Seok H, Park SJ, Chang JS, Pothoulakis C, Lamont JT, Kim H (2010) Clostridium difficile toxin A decreases acetylation of tubulin, leading to microtubule depolymerization through activation of histone deacetylase 6 , and this mediates acute inflammation. J Biol Chem 285(43):3288832896. https://doi.org/10.1074/jbc.M110.162743

30. Chumbler NM, Farrow MA, Lapierre LA, Franklin JL, Haslam $\mathrm{DB}$, Goldenring JR, et al. Correction: Clostridium difficile toxin B causes epithelial cell necrosis through an autoprocessing-independent mechanism. PLoS Pathog 2012. 8(12):https://doi.org/10. 1371/annotation/f9017013-88c8-44db-818b-08b9322f3814

Publisher's note Springer Nature remains neutral with regard to jurisdictional claims in published maps and institutional affiliations. 